\title{
Poisson Generalized Gamma Process and Its Properties
}

\author{
Ji Hwan Cha \\ Department of Statistics, Ewha Womans University \\ Seoul, 120-750, Rep. of Korea \\ e-mail: jhcha@ewha.ac.kr \\ Sophie Mercier \\ Université de Pau et des Pays de l'Adour \\ Laboratoire de Mathématiques et de leurs Applications - Pau, UMR CNRS 5142 \\ 64000, Pau, France \\ e-mail: sophie.mercier@univ-pau.fr
}

\begin{abstract}
Although the nonhomogeneous Poisson process has been intensively applied in practice, it has also its own limitations. In this paper, a new counting process model (called Poisson Generalized Gamma Process) is developed to overcome the limitations of the nonhomogeneous Poisson process. Initially, some basic stochastic properties are derived. It will be seen that this new counting process model includes both the generalized Pólya and Poisson Lindley processes as special cases. The influence of the model parameters on the behavior of the new counting process model is analyzed. The increments of the new process are shown to exhibit positive dependence properties. The corresponding compound process is defined and studied as well.
\end{abstract}

Keywords: Poisson generalized gamma process; stochastic properties; restarting property; generalized Pólya process; positive dependence

2010 Mathematics Subject Classification: Primary 60K10

Secondary 62P30

\section{Introduction}

In practice, the most typical counting process models for modeling random recurrent events are the renewal process and the nonhomogeneous Poisson process (NHPP), including the homogeneous Poisson process (HPP) as a special case in both models. The HPP can be characterized by the independent and identically distributed inter arrival 
times following an exponential distribution. The most important feature of the HPP is that it possesses both the independent and stationary increments properties. In a renewal process, the inter arrival times are independent and identically distributed according to an arbitrary distribution having a nonnegative support (Cox (1962)). In the NHPP, the stationary increments property of the HPP is dropped, but the independent increments property is kept. This allows explicit results in many applications (Cha and Finkelstein $(2009,2011 \mathrm{a})$ ), which is one of the important merits of the NHPP.

Until now, there has been much effort to generalize those basic counting processes to more general ones. For example, the compound, filtered, two dimensional, and marked Poisson processes generalize the HPP or the NHPP (see Kao, 1997). Recently, semiMarkov processes are also intensively studied and employed as one of the generalized counting processes in many applications (see, Limnios and Oprişan, 2001; Barbu and Limnios, 2008).

As mentioned in Cha (2019), the NHPP has also critical limitations which can be restrictive in various practical applications. The first critical limitation is that the variance and the mean of the number of events in $(0, t]$ are equal: $\operatorname{Var}[N(t)]=E[N(t)]$. Due to this, the NHPP is not suitable for situations where the observations are over or under dispersed. Another critical limitation is its independent increments property, which can be too restrictive to describe real life problems. For instance, in reliability applications for repairable systems, the number of failure events in the future time interval often depends on the failure event history in the past time interval and, in this case, the independent increments property obviously does not hold.

The aim of this paper is to develop a 'mathematically tractable' new counting process model, which overcomes the previous limitations of the NHPP. The mathematical tractability of the new model is a very important feature, as it allows explicit expression of the likelihood function in estimation procedure for instance and, accordingly, it makes the developed model practically useful for the applications. A first mathematically tractable extension of the NHPP called Poisson Lindley process was already proposed in Cha (2019). It is constructed as a mixed non homogeneous Poisson process with a Lindley mixing distribution. Though it addresses the previous limitations of the NHPP, the Lindley distribution has one single parameter and it is not that much flexible. This might make the Poisson Lindley process too restrictive for some applications. We here suggest to consider a more general model which encompasses the Poisson Lindley process. The approach employed in this paper is as follows. The new process is constructed as a mixed Poisson process with a generalized gamma mixing distribution. Then, based on this construction, some basic properties of the process (such as the distribution of the number of events on some time intervals) are derived. When the only available observation is the number of events occurred in some time intervals, the likelihood function can easily be derived from such results. Furthermore, the characterization of the process is provided in terms of its stochastic intensity. This stochastic intensity would allow the construction of the likelihood function based on the complete observation of the process in some time interval(s). The generalized gamma distribution encompasses the Lindley distribution and thus the new model allows to enlarge the possible behavior of the process when compared to a Poisson Lindley process, while remaining quite tractable from a mathematical point of view. As we will see, it also encompasses the general- 
ized Pólya process in Cha (2014). It hence appears as a new flexible tool for modeling random recurrent events in various practical applications, which encompasses several models from the previous literature and could be used instead of the NHPP, wherever it is not appropriate due to its limitations.

The paper is organized as follows. In Section 2, we suggest the definition of the new counting process model. The distributions for the number(s) of events in some time interval(s) are derived, together with the corresponding moment generating function and moments. In addition, the influence of the model parameters on the properties of the new counting process model is studied. In Section 3, the stochastic intensity of the counting process model is given, which allows to see that the new counting process model includes the generalized Pólya process as a special case. Furthermore, the dependence structure of the counting process model is analyzed. In Section 4, the corresponding compound process is defined and studied.

\section{Poisson Generalized Gamma Process and Its Basic Prop- erties}

To develop the new counting process model, we first recall the generalized gamma distribution introduced by Agarwal and Kalla (1996) and Ghitany (1998). A random variable $\Phi$ is said to follow the generalized gamma distribution (GGD) with parameters $(\nu, k, \alpha, l)$, where $\nu \geq 0, k, \alpha, l>0$, if its probability density function (pdf) is given by

$$
f(\phi)=\frac{\alpha^{k-\nu}}{\Gamma_{\nu}(k, \alpha l)} \frac{\phi^{k-1} \exp \{-\alpha \phi\}}{(\phi+l)^{\nu}}, \phi>0
$$

where

$$
\Gamma_{\nu}(k, \beta)=\int_{0}^{\infty} \frac{y^{k-1} \exp \{-y\}}{(y+\beta)^{\nu}} d y
$$

for all $\beta>0$, with

$$
\Gamma_{\nu}(k, \alpha l)=\int_{0}^{\infty} \frac{y^{k-1} \exp \{-y\}}{(y+\alpha l)^{\nu}} d y=\int_{0}^{\infty} \frac{\alpha^{k-\nu} y^{k-1} \exp \{-\alpha y\}}{(y+l)^{\nu}} d y .
$$

The function $\Gamma_{\nu}(k, \beta)$ is called the generalized gamma function (see Kobayashi (1991)) and if $\nu=0$, then

$$
\Gamma_{0}(k, \beta)=\int_{0}^{\infty} y^{k-1} \exp \{-y\} d y=\Gamma(k), \forall k>0 .
$$

Thus, when $\nu=0$, it can be seen that the pdf in (1) becomes that of a gamma distribution with parameter $(k, \alpha)$. In the same way, considering $l=1, \nu=-1$ and $k=1$, it is easy to check that we recover the pdf of a Lindley distribution with parameter $\alpha$. Hence, the GGD includes both the gamma and Lindley distributions as special cases.

Coming back to the general case, one can note from Gupta \& Ong (2004) that

$$
\Gamma_{\nu}(k, \beta)=\frac{\Gamma(k)}{\beta^{\nu-k}} \varphi(k, k-\nu+1 ; \beta)
$$


where

$$
\varphi(a, c ; x)=\frac{1}{\Gamma(a)} \int_{0}^{\infty} \frac{e^{-x t} t^{a-1}}{(1+t)^{a-c+1}} d t
$$

is the confluent hypergeometric function of the second kind. This allows an easy computation of $\Gamma_{\nu}(k, \beta)$, as $\varphi(a, c ; x)$ and $\Gamma(k)$ are implemented in most statistical or mathematical software.

Finally, we recall from Ghitany (1998) that the moment generating function of $\Phi$ is

$$
M_{\Phi}(s)=E\left(e^{s \Phi}\right)=\left(1-\frac{s}{\alpha}\right)^{\nu-k} \frac{\Gamma_{\nu}(k,(\alpha-s) l)}{\left.\Gamma_{\nu}(k, \alpha l)\right)}, s<\alpha,
$$

and that its $r$-th moment about the origin is

$$
E\left(\Phi^{r}\right)=\alpha^{-r} \frac{\Gamma_{\nu}(k+r, \alpha l)}{\left.\Gamma_{\nu}(k, l \alpha)\right)}, r \in \mathbb{N}^{*} .
$$

Now, we will define the new counting process model using the GGD. Let $\{M(t), t \geq$ $0\}$ be an orderly counting process. Throughout this paper, as in Cha (2019), we shall use the notation

$$
\{M(t), t \geq 0\} \sim \mathcal{N} \mathcal{H} \mathcal{P P}(\eta(t))
$$

to indicate that the counting process $\{M(t), t \geq 0\}$ follows the NHPP with intensity function $\eta(t)$. Furthermore, we shall use the notation $\Phi \sim \mathcal{G G}(\nu, k, \alpha, l)$ to represent that the continuous random variable $\Phi$ follows the GGD with parameters $(\nu, k, \alpha, l)$. In the following definition, we define the Poisson generalized gamma process (PGGP).

Definition 1. (Poisson Generalized Gamma Process) A counting process $\{N(t), t \geq$ $0\}$ is called the Poisson generalized gamma process (PGGP) with the set of parameters $(\lambda(t), \nu, k, \alpha, l), \lambda(t)>0, \forall t \geq 0, \nu \geq 0, k, \alpha, l>0$, if

(i) $\{N(t), t \geq 0\} \mid(\Phi=\phi) \sim \mathcal{N H \mathcal { P P }}(\phi \lambda(t))$;

(ii) $\Phi \sim \mathcal{G G}(\nu, k, \alpha, l)$.

A Poisson generalized gamma process hence is a mixed Poisson process with a generalized gamma mixing distribution. Thus, it could be understood as a Poisson process having a random intensity function which is a product of the deterministic intensity function and a random variable. Throughout this paper, the PGGP with the set of parameters $(\lambda(t), \nu, k, \alpha, l)$ will be denoted by $\operatorname{PGGP}(\lambda(t), \nu, k, \alpha, l)$ and we define $\Lambda(t) \equiv \int_{0}^{t} \lambda(x) d x$, $t \geq 0$. Now we will derive some basic properties of $\operatorname{PGGP}(\lambda(t), \nu, k, \alpha, l)$.

\section{Proposition 1.}

(i) $\{M(t), t \geq 0\}$ is a PGGP(1, $, k, \alpha, l)$ if and only if $\{N(t)=M(\Lambda(t)), t \geq 0\}$ is a $P G G P(\lambda(t), \nu, k, \alpha, l)$.

(ii) For $c>0$, let $\tilde{\lambda}(t)=\lambda(t) / c, \tilde{\alpha}=\alpha c$ and $\tilde{l}=l / c$. Then, a $P G G P(\tilde{\lambda}(t), \nu, k, \tilde{\alpha}, \tilde{l})$ is a $\operatorname{PGGP}(\lambda(t), \nu, k, \alpha, l)$.

Proof.

(i) Let us observe that a counting process $\{m(t), t \geq 0\}$ is a $N H P P(\phi)$ if and only if $\{n(t)=m(\Lambda(t)), t \geq 0\}$ is a $\operatorname{NHPP}(\phi \lambda(t))$. Hence $\{M(t), t \geq 0\} \mid(\Phi=\phi)$ is a 
$N H P P(\phi)$ if and only if $\{N(t)=M(\Lambda(t)), t \geq 0\} \mid(\Phi=\phi)$ is a $N H P P(\phi \lambda(t))$, which provides the result, based on the definition of a PGGP.

(ii) $\operatorname{A~} \operatorname{PGGP}(\tilde{\lambda}(t), \nu, k, \tilde{\alpha}, \tilde{l})$ is such that $\{\tilde{N}(t), t \geq 0\} \mid(\tilde{\Phi}=\tilde{\phi}) \sim N H P P(\tilde{\phi} \tilde{\lambda}(t))$ and $\tilde{\Phi} \sim \mathcal{G} \mathcal{G}(\nu, k, \tilde{\alpha}, \tilde{l})$. Now, it is known from Ghitany $(1998)$ that $c \tilde{\Phi} \sim \mathcal{G} \mathcal{G}(\nu, k, \tilde{\alpha} / c, c \tilde{l})$, that is $c \tilde{\Phi} \sim \mathcal{G} \mathcal{G}(\nu, k, \alpha, l)$. Setting $\tilde{\phi}=c \phi$ and $\Phi=c \tilde{\Phi}$, and noting that $\tilde{\phi} \tilde{\lambda}(t)=\phi \lambda(t)$, we derive that $\{\tilde{N}(t), t \geq 0\} \mid(\Phi=\phi) \sim N H P P(\phi \lambda(t))$ and $\Phi \sim \mathcal{G G}(\nu, k, \alpha, l)$, which achieves this proof.

Remark 1. Based on the second point of the previous proposition, the PGGP model as given in Definition 1 is not identifiable. Hence, whenever we are concerned with statistical issues (which is not the point of the present paper), an additional constraint should be added. A possibility (among others) might be to consider that $l=1$, as a $\operatorname{PGGP}(\lambda(t) / l, \nu, k, \alpha l, 1)$ is just a $\operatorname{PGGP}(\lambda(t), \nu, k, \alpha, l)$.

When dealing with a counting process model, one may be interested in the distributions of the number(s) of events in some time interval(s), as provided in the following theorem. The proof follows the same steps as in Proposition 1 in Cha (2019) and it is omitted.

Theorem 1. Let $\{N(t), t \geq 0\}$ be the $P G G P(\lambda(t), \nu, k, \alpha, l)$. Then, for $t>0$ and $0 \equiv t_{0}<t_{1}<t_{2}<\cdots<t_{m}$, the following properties hold:

$$
\begin{aligned}
& P(N(t)=n)=\frac{\alpha^{k-\nu}}{(\alpha+\Lambda(t))^{k+n-\nu}} \frac{\Gamma_{\nu}(k+n,(\alpha+\Lambda(t)) l)}{\Gamma_{\nu}(k, \alpha l)} \frac{\Lambda(t)^{n}}{n !} . \\
& P\left(N\left(t_{2}\right)-N\left(t_{1}\right)=n\right) \\
& =\frac{\alpha^{k-\nu}}{\left(\alpha+\left(\Lambda\left(t_{2}\right)-\Lambda\left(t_{1}\right)\right)\right)^{k+n-\nu}} \frac{\Gamma_{\nu}\left(k+n,\left(\alpha+\left(\Lambda\left(t_{2}\right)-\Lambda\left(t_{1}\right)\right)\right) l\right)}{\Gamma_{\nu}(k, \alpha l)} \frac{\left(\Lambda\left(t_{2}\right)-\Lambda\left(t_{1}\right)\right)^{n}}{n !} . \\
& P\left(N\left(t_{i}\right)-N\left(t_{i-1}\right)=n_{i}, i=1,2, \cdots, m\right) \\
& =\frac{\alpha^{k-\nu}}{\left(\alpha+\sum_{i=1}^{m}\left(\Lambda\left(t_{i}\right)-\Lambda\left(t_{i-1}\right)\right)\right)^{k+\sum_{i=1}^{m} n_{i}-\nu}} \\
& \quad \times \frac{\Gamma_{\nu}\left(k+\sum_{i=1}^{m} n_{i},\left(\alpha+\sum_{i=1}^{m}\left(\Lambda\left(t_{i}\right)-\Lambda\left(t_{i-1}\right)\right)\right) l\right)}{\Gamma_{\nu}(k, \alpha l)}\left[\prod_{i=1}^{m} \frac{\left(\Lambda\left(t_{i}\right)-\Lambda\left(t_{i-1}\right)\right)^{n_{i}}}{n_{i} !}\right] .
\end{aligned}
$$

In most practical applications, the moments of $N(t)$ are of great importance, which are given in the following theorem. In the following, the notation $M_{N(t)}(s) \equiv E\left[e^{s N(t)}\right]$ stands for the moment generating function of $N(t)$. Note that similar results are provided in Cha (2019) for points (i) and (iii) in the specific case of a Poisson Lindley process, with not exactly the same arguments for point (iii) however. We hence prefer providing the proofs of all the three points here, for sake of clarity and completeness.

Theorem 2. Let $\{N(t), t \geq 0\}$ be the $P G G P(\lambda(t), \nu, k, \alpha, l)$. Then the following properties hold.

(i) The moment generating function of $N(t)$ is given by

$$
M_{N(t)}(s)=\left(1-\Lambda(t) \frac{e^{s}-1}{\alpha}\right)^{\nu-k} \frac{\Gamma_{\nu}\left[k,\left(\alpha-\Lambda(t)\left(e^{s}-1\right)\right) l\right]}{\Gamma_{\nu}(k, \alpha l)}, s<\ln \left(\frac{\alpha+\Lambda(t)}{\Lambda(t)}\right) .
$$


(ii) The r-th moment about zero of $N(t)$ is

$$
E\left[N(t)^{r}\right]=\sum_{i=1}^{r}\left(\frac{\Lambda(t)}{\alpha}\right)^{i} \frac{\Gamma_{\nu}(k+i, \alpha l)}{\left.\Gamma_{\nu}(k, \alpha l)\right)}\left\{\begin{array}{l}
r \\
i
\end{array}\right\}, r \in \mathbb{N}^{*}
$$

where the $\{$ braces\} denote Stirling numbers of the second kind.

(iii) The mean and variance of $N(t)$ are given by

$$
E[N(t)]=\frac{\Lambda(t)}{\alpha} \frac{\Gamma_{\nu}(k+1, \alpha l)}{\Gamma_{\nu}(k, \alpha l)}
$$

and

$$
\operatorname{Var}[N(t)]=\frac{\Lambda(t)}{\alpha} \frac{\Gamma_{\nu}(k+1, \alpha l)}{\Gamma_{\nu}(k, \alpha l)}+\left(\frac{\Lambda(t)}{\alpha}\right)^{2}\left[\frac{\Gamma_{\nu}(k+2, \alpha l)}{\Gamma_{\nu}(k, \alpha l)}-\left(\frac{\Gamma_{\nu}(k+1, \alpha l)}{\Gamma_{\nu}(k, \alpha l)}\right)^{2}\right]
$$

\section{Proof.}

(i) Conditioning by $\Phi$, we have:

$$
M_{N(t)}(s)=E\left[E\left(e^{s N(t)} \mid \Phi\right)\right]
$$

where $[N(t) \mid \Phi=\phi]$ is Poisson distributed with parameter $\Lambda(t) \phi$. Based on the moment generating function of a Poisson distribution, see, e.g., Ross (2003), we derive:

$$
\begin{aligned}
M_{N(t)}(s) & =E\left[\exp \left(\Lambda(t)\left(e^{s}-1\right) \Phi\right)\right] \\
& =M_{\Phi}\left[\Lambda(t)\left(e^{s}-1\right)\right] \\
& =\left(1-\Lambda(t) \frac{e^{s}-1}{\alpha}\right)^{\nu-k} \frac{\Gamma_{\nu}\left[k,\left(\alpha-\Lambda(t)\left(e^{s}-1\right)\right) l\right]}{\Gamma_{\nu}(k, \alpha l)}
\end{aligned}
$$

for all $s<\ln \left(\frac{\alpha+\Lambda(t)}{\Lambda(t)}\right)$, due to $(3)$.

(ii) Using a similar procedure, we have:

$$
E\left[N(t)^{r}\right]=E\left[E\left(N(t)^{r} \mid \Phi\right)\right]=\sum_{i=1}^{r} E\left[(\Lambda(t) \Phi)^{i}\right]\left\{\begin{array}{l}
r \\
i
\end{array}\right\}
$$

see Riordan (1937) for the previous expression for moments of Poisson distributions with respect to Stirling numbers of the second kind.

Using (4), we derive

$$
E\left[N(t)^{r}\right]=\sum_{i=0}^{r}\left(\frac{\Lambda(t)}{\alpha}\right)^{i} \frac{\Gamma_{\nu}(k+i, \alpha l)}{\left.\Gamma_{\nu}(k, \alpha l)\right)}\left\{\begin{array}{l}
r \\
i
\end{array}\right\}
$$

(iii) The expression of $E[N(t)]$ is clear from the previous point. As for the variance, it is easily derived from

$$
\operatorname{Var}[N(t)]=E\left[N(t)^{2}\right]-(E[N(t)])^{2}
$$


In the following proposition, we compare the expectation and variance of $N(t)$ under a more general setting, when $\Phi$ follows a general distribution. The proof follows the same arguments as in Cha (2019) and it is omitted.

Proposition 2. For any mixed Poisson process $\{N(t), t \geq 0\}, \operatorname{Var}[N(t)] \geq E[N(t)]$.

Thus, we can see that the PGGP would be suitable for the cases where the observations are overdispersed with respect to a Poisson process case, for which the mean is equal to the variance.

We next investigate the influence of the parameter sets of the generalized gamma distribution on some properties of the number of events $N(t)$ in a PGGP. Let us first recall that a non negative continuous random variable $Z$ with pdf $f_{Z}$ and survival function $\bar{F}_{Z}$ is said to be Increasing (Decreasing) Hazard Rate (IHR/DHR) as soon as its hazard rate function

$$
h_{Z}(z)=\frac{f_{Z}(z)}{\bar{F}_{Z}(z)}, z \in \mathbb{R}_{+} \text {such that } \bar{F}_{Z}(z)>0,
$$

decreases (increases) with $z$. Also, it is said to be upside-down bathtub shaped when $h_{Z}(z)$ first increases and next decreases. In the same way, a discrete random variable $M$ with range $\mathbb{N}$ is said to be Increasing (Decreasing) Hazard Rate (IHR/DHR) as soon as

$$
H_{M}(n)=\frac{P(M \geq n+1)}{P(M \geq n)}=1-\frac{P(M=n)}{P(M \geq n)}, n \in \mathbb{N}
$$

decreases (increases) with $n$. It is said to be upside-down bathtub shaped when $H_{M}(z)$ first increases and next decreases. See Lai \& Xie (2006) for more details.

It is well-known that any Poisson random variable is IHR, see Barlow \& Proschan (1996). This however is not always the case for a mixed Poisson generalized gamma distribution, as shown in the next proposition.

Proposition 3. Let $\{N(t), t \geq 0\}$ be a $P G G P(\lambda(t), \nu, k, \alpha, l)$. We have the following results:

(i) If $0 \leq \nu \leq k-1$, then $N(t)$ is IHR;

(ii) If $k \leq 1$, then $N(t)$ is $D H R$;

(iii) If $\nu>k-1>0$, then $N(t)$ is upside-down bathtub shaped, that is $H_{N(t)}(n)$ first increases and next decreases, where $H_{N(t)}(n)$ is defined in a similar way as $H_{M}$ in (5).

Proof. Based on Proposition 7.2. in Grandell (1997), we know that $N(t)$ is IFR (DFR) as soon as $\Phi \sim \mathcal{G} \mathcal{G}(\lambda(t), \nu, k, \alpha, l)$ is IFR (DFR). Also, due to Theorem 4 in Ghitany (1998), the random variable $\Phi$ is IFR (resp. DFR) as soon as $0 \leq \nu \leq k-1$ (resp. $k \leq 1)$. This allows to conclude for points (i) and (ii).

For point (iii), remember from Theorem 4 in Ghitany (1998) that $\Phi$ is upside-down bathtub shaped when $\nu>k-1>0$. Following similar steps as in the proof of Proposition 7.2. in Grandell (1997) allows to conclude that the same property holds for $N(t)$, which 
concludes the proof.

Remark 2. Considering $l=1, \nu=-1$ and $k=1$, the previous proposition shows that, in case of a Poisson Lindley process, $N(t)$ always is IHR. The extension to a PGGP hence allows to enlarge the possible behavior of the process, with respect to a Poisson Lindley process.

We now introduce some basic properties of stochastic orders for further characterization of the PGGP. In the following, we denote by $X_{1} \leq_{s t} X_{2}, X_{1} \leq_{f r} X_{2}$ and $X_{1} \leq_{l r} X_{2}$ the usual stochastic order, the failure rate order and the likelihood ratio order between two random variables $X_{1}$ and $X_{2}$, respectively. The definitions for these concepts can be found, e.g., in Shaked and Shanthikumar (2007).

Lemma 1 (Shaked and Shanthikumar, 2007).

(i) If $X_{1} \leq_{s t} X_{2}$ and $g(\cdot)$ is any increasing (decreasing) function, then $E\left[g\left(X_{1}\right)\right] \leq(\geq$ ) $E\left[g\left(X_{2}\right)\right]$.

(ii) If $X_{1}$ and $X_{2}$ are two nonnegative random variables, then the following holds:

$$
X_{1} \leq_{l r} X_{2} \Rightarrow X_{1} \leq_{f r} X_{2} \Rightarrow X_{1} \leq_{s t} X_{2} \Rightarrow E\left[X_{1}\right] \leq E\left[X_{2}\right] .
$$

Proposition 4. Let $\{N(t), t \geq 0\}$ be a $\operatorname{PGGP}(\lambda(t), \nu, k, \alpha, l)$. Then, $N(t)$ increases in the likelihood ordering when $t$ increases.

Proof. Based on point (i) in Proposition 1 and Remark 1, it is enough to show the result when $\lambda(t) \equiv 1$ and $l=1$.

Let $t_{1}<t_{2}$. The aim is to show that $N\left(t_{1}\right) \leq_{l r} N\left(t_{2}\right)$, namely that

$$
g(n)=\frac{P\left(N\left(t_{2}\right)=n\right)}{P\left(N\left(t_{1}\right)=n\right)}
$$

increases with respect to $n$. Based on point (i) in Theorem 1, we may write

$$
g(n)=K\left(\frac{\frac{\alpha}{t_{1}}+1}{\frac{\alpha}{t_{2}}+1}\right)^{n} \frac{\Gamma_{\nu}\left(k+n, \alpha+t_{2}\right)}{\Gamma_{\nu}\left(k+n, \alpha+t_{1}\right)} .
$$

where $K$ is a constant. The term $\left[\left(\alpha / t_{1}+1\right) /\left(\alpha / t_{2}+1\right)\right]^{n}$ increases with respect to $n$ because $t_{1}<t_{2}$. It is hence enough to show that

$$
h(n)=\frac{\Gamma_{\nu}\left(n, \alpha_{2}\right)}{\Gamma_{\nu}\left(n, \alpha_{1}\right)}
$$

increases with respect to $n$ whenever $\alpha_{1}<\alpha_{2}$. Considering $n \in \mathbb{R}_{+}^{*}$, the sign of $h^{\prime}(n)$ is 
the same as that of

$$
\begin{aligned}
\kappa(n) & =\left(\int_{0}^{\infty} \frac{y_{1}^{n-1} e^{-y_{1}}}{\left(y_{1}+\alpha_{1}\right)^{\nu}} d y_{1}\right)\left(\int_{0}^{\infty} \frac{\ln \left(y_{2}\right) y_{2}^{n-1} e^{-y_{2}}}{\left(y_{2}+\alpha_{2}\right)^{\nu}} d y_{2}\right) \\
& -\left(\int_{0}^{\infty} \frac{\ln \left(y_{1}\right) y_{1}^{n-1} e^{-y_{1}}}{\left(y_{1}+\alpha_{1}\right)^{\nu}} d y_{1}\right)\left(\int_{0}^{\infty} \frac{y_{2}^{n-1} e^{-y_{2}}}{\left(y_{2}+\alpha_{2}\right)^{\nu}} d y_{2}\right) \\
& =\iint_{\mathbb{R}_{+}^{2}}\left(y_{1} y_{2}\right)^{n-1} e^{-\left(y_{1}+y_{2}\right)} \frac{\ln \left(y_{2}\right)-\ln \left(y_{1}\right)}{\left(y_{1}+\alpha_{1}\right)^{\nu}\left(y_{2}+\alpha_{2}\right)^{\nu}} d y_{1} d y_{2} \\
& =\kappa_{1}(n)+\kappa_{2}(n)
\end{aligned}
$$

where

$$
\begin{aligned}
& \kappa_{1}(n)=\iint_{\left\{y_{2}>y_{1}\right\}}\left(y_{1} y_{2}\right)^{n-1} e^{-\left(y_{1}+y_{2}\right)} \frac{\ln \left(y_{2}\right)-\ln \left(y_{1}\right)}{\left(y_{1}+\alpha_{1}\right)^{\nu}\left(y_{2}+\alpha_{2}\right)^{\nu}} d y_{1} d y_{2}, \\
& \kappa_{2}(n)=\iint_{\left\{y_{2}<y_{1}\right\}}\left(y_{1} y_{2}\right)^{n-1} e^{-\left(y_{1}+y_{2}\right)} \frac{\ln \left(y_{2}\right)-\ln \left(y_{1}\right)}{\left(y_{1}+\alpha_{1}\right)^{\nu}\left(y_{2}+\alpha_{2}\right)^{\nu}} d y_{1} d y_{2} .
\end{aligned}
$$

Now switching $y_{1}$ and $y_{2}$ in $\kappa_{2}(n)$, we get

$$
\kappa_{2}(n)=\iint_{\left\{y_{2}>y_{1}\right\}}\left(y_{1} y_{2}\right)^{n-1} e^{-\left(y_{1}+y_{2}\right)} \frac{\ln \left(y_{1}\right)-\ln \left(y_{2}\right)}{\left(y_{2}+\alpha_{1}\right)^{\nu}\left(y_{1}+\alpha_{2}\right)^{\nu}} d y_{1} d y_{2} .
$$

Gathering $\kappa_{1}(n)$ and $\kappa_{2}(n)$, we derive

$$
\begin{aligned}
\kappa(n)=\iint_{\left\{y_{2}>y_{1}\right\}} & \left(y_{1} y_{2}\right)^{n-1} e^{-\left(y_{1}+y_{2}\right)}\left(\ln \left(y_{2}\right)-\ln \left(y_{1}\right)\right) \\
& \times\left(\frac{1}{\left(y_{1}+\alpha_{1}\right)^{\nu}\left(y_{2}+\alpha_{2}\right)^{\nu}}-\frac{1}{\left(y_{2}+\alpha_{1}\right)^{\nu}\left(y_{1}+\alpha_{2}\right)^{\nu}}\right) d y_{1} d y_{2} .
\end{aligned}
$$

Now observe that

$$
\frac{y_{1}+\alpha_{1}}{y_{2}+\alpha_{1}}=\frac{y_{1}-y_{2}}{y_{2}+\alpha_{1}}+1 \leq \frac{y_{1}-y_{2}}{y_{2}+\alpha_{2}}+1=\frac{y_{1}+\alpha_{2}}{y_{2}+\alpha_{2}}
$$

due to $\alpha_{1}<\alpha_{2}$ and $y_{2}>y_{1}$. This entails that the term between brackets in the second line of (6) is non negative. Hence the whole integrand in (6) is non negative and $\kappa(n) \geq 0$. This shows that $h(n)$ increases with respect to $n$ and achieves the proof.

Proposition 5. Let $\left\{N_{i}(t), t \geq 0\right\}$ be a $P G G P\left(\lambda_{i}(t), \nu_{i}, k_{i}, \alpha_{i}, 1\right)$ for $i \in\{1,2\}$, where we take $l_{i}=1$, as is allowed from Remark 1 .

Assume that $\Lambda_{2}(t) \leq \Lambda_{1}(t)$ for all $t \geq 0$ and that one of the following points is true:

- $\alpha_{1}=\alpha_{2}, k_{1} \geq k_{2}$ and $k_{1}-k_{2} \geq \nu_{1}-\nu_{2}$;

- $\alpha_{1}<\alpha_{2}$ and $\left(\alpha_{2}-\alpha_{1}+k_{1}-k_{2}+\nu_{2}-\nu_{1}\right)^{2}-4\left(\alpha_{2}-\alpha_{1}\right)\left(k_{1}-k_{2}\right) \leq 0$;

- $\alpha_{1}<\alpha_{2}$ and $\alpha_{2}-\alpha_{1}+k_{1}-k_{2}+\nu_{2}-\nu_{1} \geq 0$. 
Then $N_{2}(t) \leq_{l r} N_{1}(t)$ for all $t \geq 0$.

Proof. Let us first show that each of the two points in the proposition implies that $\Phi_{2} \leq_{l r} \Phi_{1}$.

Let $f_{i}(\phi)$ be the pdf of $\Phi_{i} \sim \mathcal{G G}\left(\nu_{i}, k_{i}, \alpha_{i}, 1\right)$. Then

$$
h(\phi)=\frac{f_{1}(\phi)}{f_{2}(\phi)}=K \phi^{k}(\phi+1)^{\nu} e^{\alpha \phi},
$$

where $K$ is a positive constant, $k=k_{1}-k_{2}, \nu=\nu_{2}-\nu_{1}$ and $\alpha=\alpha_{2}-\alpha_{1}$.

We have

$$
h^{\prime}(\phi)=K\left(\alpha \phi^{2}+(\alpha+k+\nu) \phi+k\right) \phi^{k-1}(\phi+1)^{\nu-1} e^{\alpha \phi}
$$

so that $h^{\prime}(\phi)$ and $\alpha \phi^{2}+(\alpha+k+\nu) \phi+k$ share the same sign.

Now, if $\alpha=0, k+\nu \geq 0$ and $k \geq 0, h^{\prime}(\phi)$ is non negative for all $\phi$. Note that this property is not possible if $\alpha<0$.

Assume $\alpha>0$. Then $h^{\prime}(\phi)$ is non negative for all $\phi$ if $(\alpha+k+\nu)^{2}-4 \alpha k \leq 0$ or if $(\alpha+k+\nu)^{2}-4 \alpha k>0$ and

$$
-(\alpha+k+\nu)+\sqrt{(\alpha+k+\nu)^{2}-4 \alpha k} \leq 0,
$$

which is equivalent to $\alpha+k+\nu \geq 0$ (as $\alpha, k>0)$.

As a summary, $h^{\prime}(\phi)$ is non negative for all $\phi$ if either one of the following points is true:

- $\alpha=0, k+\nu \geq 0$ and $k \geq 0$;

- $\alpha>0$ and $(\alpha+k+\nu)^{2}-4 \alpha k \leq 0$;

- $\alpha>0$ and $\alpha+k+\nu \geq 0$.

Note that these conditions are just equivalent to the three points in the proposition. Hence, under one of these three points, the function $h(\phi)$ is non decreasing, which implies that $\Phi_{2} \leq_{l r} \Phi_{1}$.

Now, let $\left\{M_{i}(t), t \geq 0\right\}$ be a $\operatorname{PGGP}\left(t, \nu_{i}, k_{i}, \alpha_{i}, 1\right)$ for $i \in\{1,2\}$.

Then $\left[M_{i}(t) \mid \Phi_{i}=\phi\right] \sim \operatorname{Poi}(\phi t)$ (Poisson distribution with parameter $\phi t$ ), which increases in the likelihood ordering when $\phi$ increases, see Belzunce et al. (2016) page 104. As $\Phi_{2} \leq_{l r} \Phi_{1}$, we derive from Theorem 1.C.17. in Shaked and Shanthikumar (2007) that $M_{2}(t) \leq_{l r} M_{1}(t)$ for all $t \geq 0$.

Then $M_{2}\left(\Lambda_{2}(t)\right) \leq_{l r} M_{1}\left(\Lambda_{2}(t)\right)$. Now, as $\Lambda_{2}(t) \leq \Lambda_{1}(t)$, we have $M_{1}\left(\Lambda_{2}(t)\right) \leq_{l r}$ $M_{1}\left(\Lambda_{1}(t)\right)$ from Proposition 4. Finally, we obtain $M_{2}\left(\Lambda_{2}(t)\right) \leq_{l r} M_{1}\left(\Lambda_{1}(t)\right)$, which provides the result, based on point (i) in Proposition 1.

As a by-product of the previous proposition, one can see that, if all parameters are fixed except from one, then $N(t)$ increases in the likelihood ordering when $\Lambda(t)$ or $k$ increases, or when $\nu$ or $\alpha$ decreases. 


\section{Further Properties of Poisson Generalized Gamma Pro- cess}

We now derive the stochastic intensity of the PGGP. Let $\{N(t), t \geq 0\}$ be an orderly point process and let $\mathcal{H}_{t-}$ be the $\sigma$-field generated by $\{N(u), 0 \leq u<t\}$, that is $\mathcal{H}_{t-}$ stands for the history of the process on $[0, t)$. Observe that $\mathcal{H}_{t-}$ can be equivalently defined in terms of the number of events in $[0, t)$ denoted by $N(t-)$ and the sequential arrival times of events, i.e., $0 \leq T_{1} \leq T_{2} \leq \cdots \leq T_{N(t-)}<t$. As discussed, e.g., in Cha and Finkelstein (2011b), and Cha (2014), the stochastic intensity $\lambda_{t}$ of an orderly point process $\{N(t), t \geq 0\}$ is defined as the following limit:

$$
\begin{aligned}
\lambda_{t} & \equiv \lim _{\Delta t \rightarrow 0} \frac{P\left(N(t, t+\Delta t)=1 \mid \mathcal{H}_{t-}\right)}{\Delta t} \\
& =\lim _{\Delta t \rightarrow 0} \frac{E\left[N(t, t+\Delta t) \mid \mathcal{H}_{t-}\right]}{\Delta t},
\end{aligned}
$$

where $N\left(t_{1}, t_{2}\right), t_{1}<t_{2}$, represents the number of events in $\left[t_{1}, t_{2}\right)$. Then the above stochastic intensity in (7) has the following heuristic interpretation: $\lambda_{t} d t=E\left[d N(t) \mid \mathcal{H}_{t-}\right]$, which is very similar to the ordinary failure rate or hazard rate of a random variable (Aven and Jensen (1999)). Note that a similar result as the following theorem was provided in Cha (2019) in the specific case of a Poisson Lindley process, with different (and longer) arguments however.

Theorem 3. Let $\{N(t), t \geq 0\}$ be the $P G G P(\lambda(t), \nu, k, \alpha, l)$. The stochastic intensity $\lambda_{t}$ of $\{N(t), t \geq 0\}$ is given by

$$
\lambda_{t}=\frac{1}{(\alpha+\Lambda(t))} \frac{\Gamma_{\nu}(k+N(t-)+1,(\alpha+\Lambda(t)) l)}{\Gamma_{\nu}(k+N(t-),(\alpha+\Lambda(t)) l)} \lambda(t) .
$$

Proof. Let us first consider the case of a PGGP $\{M(t), t \geq 0\}$ with parameter $\left(\lambda^{(0)}(t)=t, \nu, k, \alpha, l\right)$. In that case, it is known from Proposition 4.1 page 65 in Grandell (1997) that the corresponding stochastic intensity $\lambda_{t}^{(0)}$ is

$$
\lambda_{t}^{(0)}=\frac{\int_{0}^{\infty} \phi^{N(t-)+1} e^{-\phi t} f_{(\nu, k, \alpha, l)}(\phi) d \phi}{\int_{0}^{\infty} \phi^{N(t-)} e^{-\phi t} f_{(\nu, k, \alpha, l)}(\phi) d \phi},
$$

where $f_{(\nu, k, \alpha, l)}$ stands for the pdf of a GGD with parameters $(\nu, k, \alpha, l)$. Inserting the expression of this pdf (see (1)) into (9), we obtain

$\lambda_{t}^{(0)}=\frac{\int_{0}^{\infty} \phi^{k+N(t-)} \frac{e^{-\phi(t+\alpha)}}{(\phi+l)^{\nu}} d \phi}{\int_{0}^{\infty} \phi^{k+N(t-)-1} \frac{e^{-\phi(t+\alpha)}}{(\phi+l)^{\nu}} d \phi}=\frac{\Gamma_{\nu}(k+N(t-)+1,(\alpha+t) l)}{(\alpha+t)^{k+N(t-)+1-\nu}} \times \frac{(\alpha+t)^{k+N(t-)-\nu}}{\Gamma_{\nu}(k+N(t-),(\alpha+t) l)}$.

through normalization of the pdf of $f_{(\nu, k+N(t-)+1, \alpha+t, l)}$ and $f_{(\nu, k+N(t-), \alpha+t, l)}$, which appear in the numerator and denominator, respectively.

Now, it is known from Proposition 1, that the $\operatorname{PGGP}(\lambda(t), \nu, k, \alpha, l)$ can be recovered through setting $\{N(t)=M(\Lambda(t)), t \geq 0\}$. 
The result is next easily derived from page 79 in Grandell (1997), which implies that

$$
\lambda_{t}=\lambda_{\Lambda(t)}^{(0)} \lambda(t) .
$$

From Theorem 3, we can see that the PGGP has the Markov property, namely the conditional distribution of the process state $N(t)$ at time $t$ given its full history $\mathcal{H}_{t-}$ only depends on the last state of the process $N(t-)$. Furthermore, due to the explicit result in Theorem 3 and as briefly discussed in Remark 1 of Cha (2019) for the case of Poisson Lindley process, it is possible to construct likelihood function for the estimation of model parameters, which would be crucial for practical application of the developed model.

Based on Theorem 3, it can be shown that the PGGP includes the generalized Pólya process (GPP) studied in the literature (Cha (2014)) as a special case. To show this, we first recall the definition of the GPP here.

Definition 2. (Generalized Pólya Process) A counting process $\{N(t), t \geq 0\}$ is called the generalized Pólya process (GPP) with the set of parameters $(\kappa(t), \gamma, \tau), \gamma \geq 0$, $\tau>0$, if

(i) $N(0)=0$;

(ii) The stochastic intensity of $\{N(t), t \geq 0\}$ is given by $\lambda_{t}=(\gamma N(t-)+\tau) \kappa(t)$.

The GPP defined in Definition 2 will be denoted by $\operatorname{GPP}(\kappa(t), \gamma, \tau)$.

Proposition 6. Let $\{N(t), t \geq 0\}$ be the $P G G P(\lambda(t), \nu, k, \alpha, l)$. If we set $\lambda(t)=$ $\kappa(t) \exp \left\{\gamma \int_{0}^{t} \kappa(u) d u\right\}, t \geq 0, \nu=0, k=\tau / \gamma$, and $\alpha=1 / \gamma$, then this counting process $\{N(t), t \geq 0\}$ under this specific setting becomes the GPP $(\kappa(t), \gamma, \tau)$, regardless of $l$.

Proof.

As explained before, when $\nu=0$,

$$
\Gamma_{\nu}(k, \alpha l)=\Gamma(k) .
$$

Thus, under the specific setting with $\lambda(t)=\kappa(t) \exp \left\{\gamma \int_{0}^{t} \kappa(u) d u\right\}, t \geq 0, \nu=0$, $k=\tau / \gamma$, and $\alpha=1 / \gamma$, the stochastic intensity in (8) now becomes

$$
\begin{aligned}
\lambda_{t} & =\frac{1}{\left(1 / \gamma+1 / \gamma\left(\exp \left\{\gamma \int_{0}^{t} \kappa(u) d u\right\}-1\right)\right)} \frac{\Gamma(\tau / \gamma+N(t-)+1)}{\Gamma(\tau / \gamma+N(t-))} \kappa(t) \exp \left\{\gamma \int_{0}^{t} \kappa(u) d u\right\} \\
& =(\gamma N(t-)+\tau) \kappa(t),
\end{aligned}
$$

which completes the proof.

In the following, it will be shown that the PGGP has the so-called 'restarting property' introduced in Cha (2014).

\section{Definition 3. Restarting Property}

Let $t>0$ be an 'arbitrary' time point. If the conditional future stochastic process from $t$, given the history until time $t$, follows the same type of stochastic process with possibly different set of process parameters, then the process is said to possess the restarting 
property. A stochastic process that enjoys the restarting property is called a restarting process.

The restarting property is explained in much more details in Cha (2014), where several examples are given.

Proposition 7. Let $\{N(t), t \geq 0\}$ be $P G G P(\lambda(t), \nu, k, \alpha, l)$. At an arbitrary time $u>0$, given $\left\{N(u-)=n, T_{1}=t_{1}, T_{2}=t_{2}, \cdots, T_{n}=t_{n}\right\}$, the conditional future process $\left\{N_{u}(t), t \geq 0\right\}$, where $N_{u}(t) \equiv N(u+t)-N(u)$, is a PGGP with the set of parameters $(\lambda(u+t), \nu, k+n, \alpha+\Lambda(u), l)$.

\section{Proof.}

From (8), given $\left\{N(u-)=n, T_{1}=t_{1}, T_{2}=t_{2}, \cdots, T_{n}=t_{n}\right\}$, the stochastic intensity of the future process $\left\{N_{u}(t), t \geq 0\right\}$, denoted by $\lambda_{t}^{u}$, is given by

$$
\lambda_{t}^{u}=\frac{1}{\left(\alpha+\Lambda(u)+\Lambda_{u}(t)\right)} \frac{\Gamma_{\nu}\left(k+n+N_{u}(t-)+1,\left(\alpha+\Lambda(u)+\Lambda_{u}(t)\right) l\right)}{\Gamma_{\nu}\left(k+n+N_{u}(t-),\left(\alpha+\Lambda(u)+\Lambda_{u}(t)\right) l\right)} \lambda_{u}(t),
$$

where $\lambda_{u}(t) \equiv \lambda(u+t)$, and $\Lambda_{u}(t) \equiv \int_{0}^{t} \lambda_{u}(x) d x$. Thus, it can be seen that $\lambda_{t}^{u}$ is the stochastic intensity of PGGP with the set of parameters $(\lambda(u+t), \nu, k+n, \alpha+\Lambda(u), l)$.

Relying on Proposition 7, some conditional properties of the PGGP can be conveniently obtained. For example, let us consider $P(N(u+t)-N(u)=n \mid N(u)=m)$. From Proposition 7, given $N(u)=m$, the conditional process $\left\{N_{u}(t), t \geq 0\right\}$ is a PGGP with the set of parameters $(\lambda(u+t), \nu, k+m, \alpha+\Lambda(u), l)$. Then, from Theorem 1-(i),

$$
\begin{aligned}
P(N(u+t)-N(u)=n \mid N(u) & =m)=\frac{(\alpha+\Lambda(u))^{k+m-\nu}}{\left(\alpha+\Lambda(u)+\Lambda_{u}(t)\right)^{k+m+n-\nu}} \\
\times & \frac{\Gamma_{\nu}\left(k+m+n,\left(\alpha+\Lambda(u)+\Lambda_{u}(t)\right) l\right)}{\Gamma_{\nu}(k+m,(\alpha+\Lambda(u)) l)} \frac{\Lambda_{u}(t)^{n}}{n !} .
\end{aligned}
$$

In the following proposition, we show that $\lambda_{t}$ in (8) is increasing in $N(t-)$, implying that the proneness to the future event occurrence is increasing with the number of previously occurred events.

Proposition 8. Let $\{N(t), t \geq 0\}$ be the $P G G P(\lambda(t), \nu, k, \alpha, l)$. Then the stochastic intensity $\lambda_{t}$ of $\{N(t), t \geq 0\}$ is increasing in $N(t-)$.

\section{Proof.}

It is sufficient to show that

$$
\eta(k) \equiv \frac{\Gamma_{\nu}(k+1, \alpha l)}{\Gamma_{\nu}(k, \alpha l)}
$$

is increasing in $k>0$ for any $\nu \geq 0, \alpha, l>0$. Observe that, from the second expression in $(2)$,

$$
\eta^{\prime}(k)=\frac{1}{\Gamma_{\nu}(k, \alpha l)^{2}}\left[\int_{0}^{\infty} \frac{\ln (y) \cdot y^{k} \exp \{-y\}}{(y+\alpha l)^{\nu}} d y \cdot \int_{0}^{\infty} \frac{y^{k-1} \exp \{-y\}}{(y+\alpha l)^{\nu}} d y\right.
$$




$$
\begin{gathered}
\left.-\int_{0}^{\infty} \frac{\ln (y) \cdot y^{k-1} \exp \{-y\}}{(y+\alpha l)^{\nu}} d y \cdot \int_{0}^{\infty} \frac{y^{k} \exp \{-y\}}{(y+\alpha l)^{\nu}} d y\right] \\
=\frac{\Gamma_{\nu}(k, \alpha l) \Gamma_{\nu}(k+1, \alpha l)}{\Gamma_{\nu}(k, \alpha l)^{2}}\left[\int_{0}^{\infty} \frac{1}{\Gamma_{\nu}(k+1, \alpha l)} \frac{\ln (y) \cdot y^{k} \exp \{-y\}}{(y+\alpha l)^{\nu}} d y\right. \\
\left.-\int_{0}^{\infty} \frac{1}{\Gamma_{\nu}(k, \alpha l)} \frac{\ln (y) \cdot y^{k-1} \exp \{-y\}}{(y+\alpha l)^{\nu}} d y\right] .
\end{gathered}
$$

Denote by $Y_{1}$ the random variable which has the corresponding pdf

$$
f_{Y_{1}}(y)=\frac{1}{\Gamma_{\nu}(k, \alpha l)} \frac{y^{k-1} \exp \{-y\}}{(y+\alpha l)^{\nu}}
$$

and denote by $Y_{2}$ the random variable which has the corresponding pdf

$$
f_{Y_{2}}(y)=\frac{1}{\Gamma_{\nu}(k+1, \alpha l)} \frac{y^{k} \exp \{-y\}}{(y+\alpha l)^{\nu}}
$$

respectively. Then,

$$
\frac{f_{Y_{1}}(y)}{f_{Y_{2}}(y)}=\frac{\Gamma_{\nu}(k+1, \alpha l)}{\Gamma_{\nu}(k, \alpha l)} \frac{1}{y}
$$

which is decreasing in $y$. Then, from Definition $3, Y_{1} \leq_{l r} Y_{2}$ and, by Lemma $1, Y_{1} \leq_{s t} Y_{2}$. Furthermore, $\ln (y)$ is an increasing function of $y$. Thus, again from Lemma 1 ,

$$
\begin{aligned}
& \int_{0}^{\infty} \frac{1}{\Gamma_{\nu}(k+1, \alpha l)} \frac{\ln (y) \cdot y^{k} \exp \{-y\}}{(y+\alpha l)^{\nu}} d y=E\left[\ln \left(Y_{2}\right)\right] \\
& \geq E\left[\ln \left(Y_{1}\right)\right]=\int_{0}^{\infty} \frac{1}{\Gamma_{\nu}(k, \alpha l)} \frac{\ln (y) \cdot y^{k-1} \exp \{-y\}}{(y+\alpha l)^{\nu}} d y
\end{aligned}
$$

which implies $\eta^{\prime}(k) \geq 0, k>0$, and, accordingly, $\eta(k)$ is increasing in $k$.

As mentioned before, Proposition 8 implies that the proneness to the future event occurrence is increasing in the number of events occurred previously. This again implies a kind of positive dependent increments property. In the following, we analyze the dependence structure in the increments of PGGP. For this, we recall a concept of multivariate positive dependence for multivariate increments in a counting process model suggested in Cha (2019).

Definition 4. (Positive Upper Orthant Dependent Increments) A counting process $\{N(t), t \geq 0\}$ is said to have positive upper orthant dependent increments if for any arbitrary integer $m \geq 2$ and $0<t_{1}<t_{2}<\cdots<t_{m}$,

$$
\left.P\left(N\left(t_{i}+\Delta t_{i}\right)-N\left(t_{i}\right)>n_{i}, i=1,2, \cdots, m\right) \geq \prod_{i=1}^{m} N\left(t_{i}+\Delta t_{i}\right)-N\left(t_{i}\right)>n_{i}\right),
$$

for all $n_{i}, i=1,2, \cdots, m$, where $t_{i}+\Delta t_{i} \leq t_{i+1}, i=1,2, \cdots, m-1$. 
Theorem 4. Any mixed Poisson process has positive upper orthant dependent increments.

Proof. The proof is similar to that of Theorem 2 in Cha (2019) and it is omitted.

We now derive the conditional joint distribution of the arrival times in $(0, t]$, given $N(t), t>0$, in any mixed Poisson process.

Theorem 5. For any mixed Poisson process, the conditional joint distribution (pdf) of the arrival times

$$
\left(T_{1}, T_{2}, \cdots, T_{n} \mid N(t)=n\right)
$$

is given by

$$
f_{\left(T_{1}, T_{2}, \cdots, T_{N(t)} \mid N(t)\right)}\left(t_{1}, t_{2}, \cdots, t_{n} \mid n\right)=n ! \prod_{i=1}^{n}\left(\frac{\lambda\left(t_{i}\right)}{\Lambda(t)}\right),
$$

where $0<t_{1} \leq t_{2} \leq \cdots \leq t_{n} \leq t$.

Proof.

Note that the conditional joint arrival times distribution (pdf) in the process can be expressed as

$$
\begin{aligned}
& f_{\left(T_{1}, T_{2}, \cdots, T_{n} \mid N(t)\right)}\left(t_{1}, \cdots, t_{n} \mid n\right) \\
& =E_{(\Phi \mid N(t)=n)}\left[f_{\left(T_{1}, T_{2}, \cdots, T_{n} \mid N(t), \Phi\right)}\left(t_{1}, \cdots, t_{n} \mid n, \Phi\right)\right],
\end{aligned}
$$

where $f_{\left(T_{1}, T_{2}, \cdots, T_{n} \mid N(t), \Phi\right)}\left(t_{1}, \cdots, t_{n} \mid n, \phi\right)$ is the conditional joint distribution of

$$
\left(T_{1}, T_{2}, \cdots, T_{n} \mid N(t)=n, \Phi=\phi\right) .
$$

It is well known that the conditional arrival time distribution of $T_{1}, T_{2}, \cdots, T_{N(t)}$ in $(0, t]$, given that $N(t)=n, \Phi=\phi$, is given by (Ross, 1996)

$$
n ! \prod_{i=1}^{n}\left(\frac{\phi \lambda\left(t_{i}\right)}{\phi \Lambda(t)}\right)=n ! \prod_{i=1}^{n}\left(\frac{\lambda\left(t_{i}\right)}{\Lambda(t)}\right), 0<t_{1} \leq t_{2} \leq \cdots \leq t_{n} \leq t
$$

which is independent of $\phi$. Therefore, we now have the desired result.

It can be seen from Theorem 5 that whatever the mixing distribution is, given $N(t)=$ $n$, the $n$ first arrival times $T_{1}, T_{2}, \cdots, T_{n}$ in a mixed Poisson process have the same distribution as the order statistics corresponding to $n$ independent random variables identically distributed according to (pdf)

$$
\left(\frac{\lambda(x)}{\Lambda(t)}\right), 0<x \leq t .
$$




\section{Compound PGGP}

Let a stochastic process $\{W(t), t \geq 0\}$ be defined by

$$
W(t)=\sum_{i=1}^{N(t)} X_{i}, \quad t \geq 0,
$$

where $\{N(t), t \geq 0\}$ is the PGGP, and $\left\{X_{i}, i \geq 1\right\}$ is a family of independent and identically distributed random variables that is independent of $\{N(t), t \geq 0\}$. Then the stochastic process $\{W(t), t \geq 0\}$ is said to be a compound Poisson generalized gamma process. Various practical applications of such compound process defined in (11) can be found in Ross (2003).

Let $M_{X}(s) \equiv E\left[e^{s X_{i}}\right]$, the MGF of $X_{i}$. The following result gives the moment generating function, the mean and the variance of $W(t)$. Note that similar results were provided in Cha (2019) in the specific case of a Poisson Lindley process, with different and longer arguments however.

Theorem 6. The moment generating function of $W(t)$, which is denoted by $M_{W(t)}(s)$, is given by

$$
M_{W(t)}(s)=\frac{\alpha^{k-\nu}}{\left(\alpha+\Lambda(t)-M_{X}(s) \Lambda(t)\right)^{k-\nu}} \frac{\Gamma_{\nu}\left(k,\left(\alpha+\Lambda(t)-M_{X}(s) \Lambda(t)\right) l\right)}{\Gamma_{\nu}(k, \alpha l)},
$$

and the mean and variance of $W(t)$ are

$$
E[W(t)]=\frac{\Lambda(t) E[X]}{\alpha} \frac{\Gamma_{\nu}(k+1, \alpha l)}{\Gamma_{\nu}(k, \alpha l)}
$$

and

$$
\begin{aligned}
\operatorname{Var}[W(t)]= & \frac{\Lambda(t) E\left[X^{2}\right]}{\alpha} \frac{\Gamma_{\nu}(k+1, \alpha l)}{\Gamma_{\nu}(k, \alpha l)} \\
& +\left(\frac{\Lambda(t) E[X]}{\alpha}\right)^{2}\left[\frac{\Gamma_{\nu}(k+2, \alpha l)}{\Gamma_{\nu}(k, \alpha l)}-\left(\frac{\Gamma_{\nu}(k+1, \alpha l)}{\Gamma_{\nu}(k, \alpha l)}\right)^{2}\right] .
\end{aligned}
$$

\section{Proof.}

Given $\Phi$, the random variable $W(t)$ is known to be conditionally distributed as a compound Poisson distribution, with $\Phi \Lambda(t)$ as parameter for the Poisson distribution.

Based on the formula for the moment generating function of a compound Poisson distribution, we have:

$$
M_{W(t)}(s)=E\left[E\left(e^{s \sum_{i=1}^{N(t)} X_{i}} \mid \Phi\right)\right]=E\left[e^{\Phi \Lambda(t)\left(M_{X}(s)-1\right)}\right] .
$$

This can be written as

$$
M_{W(t)}(s)=M_{\Phi}\left[\Lambda(t)\left(M_{X}(s)-1\right)\right]
$$


where $M_{\Phi}$ is provided by (3). This easily provides the first result.

$E[W(t)]$ and $\operatorname{Var}[W(t)]$ can next be obtained by using $\left.M_{W(t)}^{\prime}(s)\right|_{s=0}$ and $\left.M_{W(t)}^{\prime \prime}(s)\right|_{s=0}$.

Proposition 9. Let $\{W(t), t \geq 0\}$ be a compound Poisson generalized gamma process, as previously described. Then we have the following results.

1. If $X_{1}$ is IHR, then $W\left(t_{1}\right) \leq_{h r} W\left(t_{2}\right)$ for all $t_{1}, t_{2} \geq 0$ such that $t_{1} \leq t_{2}$.

2. If the pdf of $X_{1}$ is log-concave, then $W\left(t_{1}\right) \leq_{l r} W\left(t_{2}\right)$ for all $t_{1}, t_{2} \geq 0$ such that $t_{1} \leq t_{2}$.

Proof. Based on Proposition 4, we have $N\left(t_{1}\right) \leq_{l r} N\left(t_{2}\right)$ for all $t_{1}, t_{2} \geq 0$ such that $t_{1} \leq t_{2}$. Theorems 1.C.11 and 1.C.12 page 47 in Shaked \& Shantikumar (2007) then allow to conclude for both points.

Remark 3. A similar result as the first point is valid assuming $X_{1}$ to have a decreasing reversed hazard rate, which leads to the conclusion that $W_{2}(t)$ is smaller than $W_{1}(t)$ in the sense of the reversed hazard rate ordering, see Shaked \& Shantikumar (2007) for more details.

Proposition 10. Let $\left\{W_{i}(t), t \geq 0\right\}, i \in\{1,2\}$ be two compound Poisson generalized gamma processes constructed from the same sequence $\left\{X_{n}, n \geq 1\right\}$ and two different $P G G P\left(\lambda_{i}(t), \nu_{i}, k_{i}, \alpha_{i}, 1\right), i \in\{1,2\}$, where we take $l_{i}=1$, as is allowed from Remark 1 . Assume that $\Lambda_{2}(t) \leq \Lambda_{1}(t)$ for all $t \geq 0$ and that one of the points from Proposition 5 is true. Then we have the following results.

1. If $X_{1}$ is IHR. then $W_{2}(t) \leq_{h r} W_{1}(t)$ for all $t \geq 0$.

2. If the pdf of $X_{1}$ is log-concave, then $W_{2}(t) \leq_{l r} W_{1}(t)$ for all $t \geq 0$.

The proof is similar to that of Proposition 9 and it is omitted. A similar remark to Remark 3 is also valid.

\section{Acknowledgments}

The authors thank the reviewers for helpful comments and advices. This work was supported by the National Research Foundation of Korea (NRF) grant funded by the Korea government (MSIP) (No. 2019R1A2B5B02069500). This work was also supported by Basic Science Research Program through the National Research Foundation of Korea (NRF) funded by the Ministry of Education (Grant Number: 2019R1A6A1A11051177).

\section{References}

Agarwal, S. K., \& Kalla, S. L. (1996). A generalized gamma distribution and its application in reliability. Communications in Statistics-Theory and Methods 25, 201-210.

Aven, T., \& Jensen, U. (1999). Stochastic models in reliability. New York: Springer. 
Barlow, R.E., \& Proschan, F., with contributions by L. Hunter (1996). Mathematical Theory of Reliability. SIAM Series in Applied Mathematics, Wiley, New York.

Barbu, V. S., \& Limnios, N. (2008). Semi-Markov chains and hidden semi-Markov models toward applications (Their use in reliability and DNA analysis). New York: Springer.

Belzunce, F., Martínez-Riquelme, C. \& Mulero, J. (2016) An introduction to stochastic orders. Elsevier/Academic Press, Amsterdam.

Cox, D. R. (1962). Renewal theory. Mondon: Methuen.

Cha, J. H. (2014). Characterization of the generalized Pólya process and its applications. Advances in Applied Probability 46, 1148-1171.

Cha, J. H. (2019). Poison Lindley process and its main properties. Statistics $\&$ Probability Letters 152, 74-81.

Cha, J. H., \& Finkelstein, M. (2009). On a terminating shock process with independent wear increments. Journal of Applied Probability 46, 353-362.

Cha, J. H., \& Finkelstein, M. (2011a). On new classes of extreme shock models and some generalizations. Journal of Applied Probability 48, 258-270.

Cha, J. H., \& Finkelstein, M. (2011b). Stochastic intensity for minimal repairs in heterogeneous populations. Journal of Applied Probability 48, 868-876.

Ghitany, M. E. (1998). On a recent generalization of gamma distribution. Communications in Statistics-Theory and Methods 27, 223-233.

Grandell, J. (1997). Mixed poisson processes. Monographs on Statistics and Applied Probability, 77. Chapman \& Hall, London.

Gupta, R. C., \& Ong, S. H. (2004). A new generalization of the negative binomial distribution. Computational statistics $\&$ data analysis 45(2), 287-300.

Kao, P. C. (1997). An Introduction to Stochastic Processes. Belmont: Duxbury Press.

Kobayashi, K. (1991). On generalized gamma functions occurring in diffraction theory. Journal of the Physical Society of Japan 60, 1501-1512.

Limnios, N., \& Oprişan, G. (2001). Semi-Markov processes and reliability. Boston: Birkhäuser.

Riordan, J. (1937). Moment recurrence relations for binomial, Poisson and hypergeometric frequency distributions. Annals of Mathematical Statistics 8(2), 103-111

Ross, S. M. (1996). Stochastic processes. New York: John Wiley.

Ross, S. M. (2003). Introduction to probability models, Eighth Edition. San Diego: Academic Press.

Shaked, M., \& Shanthikumar, J. G. (2007). Stochastic orders. New York: Springer.

Lai, C.-D. \& Xie, M. (2006). Stochastic Ageing and Dependence for Reliability. SpringerVerlag, New York. 